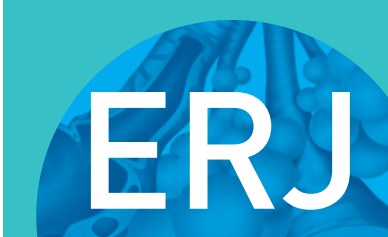

open research
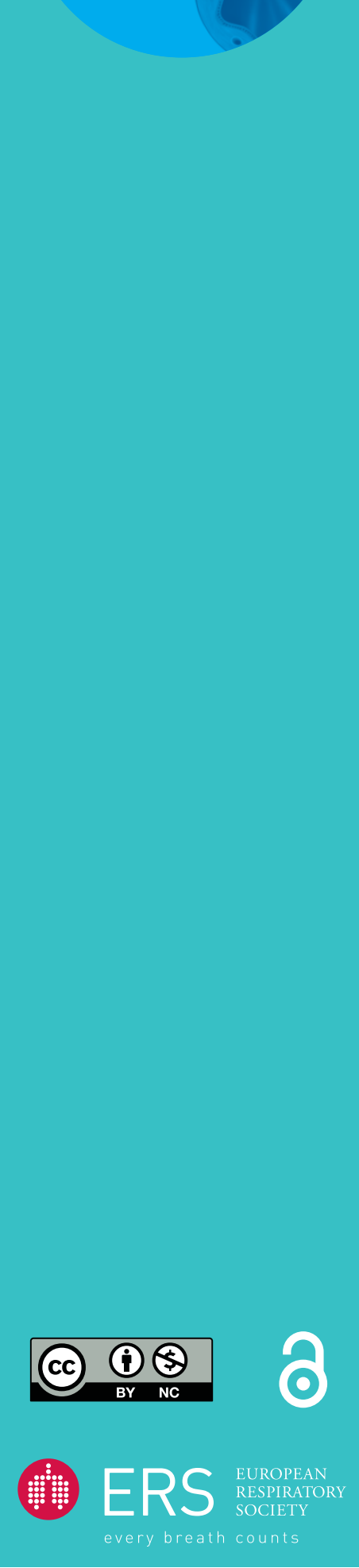

\section{Combining biopsy tools improves mutation detection rate in central} lung cancer

\section{To the Editor:}

Molecular genetic testing (such as next-generation sequencing (NGS)) and programmed death ligand 1 (PD-L1) staining have become essential for the evaluation of lung cancer tissue $[1,2]$. Endobronchial forceps biopsy (FB) is considered the gold standard for tissue sampling in central lung cancer [3].

In this prospective pilot trial, it was analysed, whether the combined analysis of $\mathrm{FB}$, endobronchial ultrasound-guided transbronchial needle aspiration (EBUS-TBNA) samples from the primary tumour (EBUS-TBNA-TU) and mediastinal lymph nodes (EBUS-TBNA-LN), and peripheral blood plasma-derived circulating tumour DNA (ctDNA) improves the detection rate of oncogenic mutations and PD-L1-positivity.

Prospective pilot trial in 30 consecutive subjects with central lung cancer (10 female; median age 66 years; 29 current/ex-smokers). The sequence of FB and EBUS-TBNA tissue sampling was randomised to avoid sampling bias. Inclusion criteria were computed tomography with suspected endobronchial tumour infiltration, indication for bronchoscopic sampling and written informed consent. The study was approved by the Ethics Commission of Cologne University's Faculty of Medicine and registered on www. clinicaltrials.gov (NCT03017183).

Flexible videobronchoscopy (BF-H190; Olympus, Tokyo, Japan) and EBUS bronchoscopy (BF-UC180F; Olympus) was performed in each subject. Biopsies were sampled by FB (FB 231D; Olympus) and EBUS-TBNA (NA-201SX-4021; Olympus).

Histological samples were analysed by standard staining protocols. PD-L1 immunohistochemistry (IHC) (Dako-22C3-pharmDx; Agilent, Santa Clara, CA, USA) was described by a five-level score [4, 5].

Areas with tumour cells were marked with a thin marker on the coverslip. Only marked areas were used for DNA extraction (QIAsymphony DNA kit; QIAGEN, Hilden, Germany). Mutational analysis was performed by massively parallel sequencing (Ion-AmpliSeq Custom DNA Panel and Ion-AmpliSeq Library Kit 2.0; Thermo-Fisher-Scientific, Waltham, MA, USA). Overall, 69 exons were analysed in every subject. Libraries were generated by adapter ligation and target enrichment (Gene-Read DNA Library I Core Kit and Gene-Read DNA I Amp Kit (QIAGEN), and NEXTflex DNA Barcodes (BioScientific, Austin, TX, USA)). 12 pmol of the constructed libraries were sequenced on the MiSeq (Illumina, San Diego, CA, USA). Computational analysis was performed to detect relevant somatic mutations in a quantitative manner [6]. Allele frequency is given in percent of mutated DNA copy numbers. NGS analysis was not performed in case of small cell lung cancer histology, as clinically useful driver mutations are rare in this tumour [7]. Fluorescence in situ hybridisation testing was performed for ALK translocation analysis [8]. ctDNA was extracted from blood plasma (QIAamp circulating nucleic acid kit; Qiagen) and was subjected to NGS.

Descriptive statistics are presented as $\mathrm{n}(\%)$ as well as median (interquartile range) where appropriate. The number of detected mutations, the median number of mutations per patient and the adjusted mutation

@ERSpublications

In central exophytic lung cancer, the detection rate of oncogenic mutations and PDL1 positivity may be increased by combined sampling by forceps and EBUS-TBNA. The additional sampling of mediastinal lymph node and ctDNA may not be of additional benefit. https://bit.ly/2Ve41EF

Cite this article as: Hagmeyer L, Schäfer S, Engels M, et al. Combining biopsy tools improves mutation detection rate in central lung cancer. ERJ Open Res 2020; 6: 00002-2020 [https://doi.org/ $10.1183 / 23120541.00002-2020]$.

Copyright $\odot$ ERS 2020. This article is open access and distributed under the terms of the Creative Commons Attribution NonCommercial Licence 4.0. 
frequency were compared between sampling techniques. In case of non-normal distribution, the Wilcoxon signed-rank test was applied to determine p-values.

EBUS-TBNA-TU and FB of the central tumour were performed in all 30 subjects. Except for one FB sample, malignancy could be detected in all samples. Sampling of lymph nodes detected malignancy in 16 out of 29 analysed cases.

The final pathomorphological classifications were nine squamous cell carcinoma (SCC); seven adenocarcinoma; six small cell lung cancer (SCLC); four non-small cell lung cancer, not otherwise specified (NSCLC/NOS); one mixed carcinoma (SCC+SCLC); one adenoid-cystic carcinoma; one typical carcinoid and one mucoepidermoid carcinoma.

Out of 30 cases, six cases with SCLC and one case with SCLC/SCC histology were not subjected to NGS. NGS data were available from 21 of the remaining 23 subjects (91\%). In two cases, EBUS-TBNA-TU and FB did not yield enough material. NGS was performed in both, EBUS-TBNA-TU and FB samples, in 20 patients, while in one case only FB provided enough material.

In these 20 subjects, overall 1380 exons have been analysed. Comparing FB and EBUS-TBNA-TU, congruent findings could be determined in about 1371 exons. By this, the accordance between both sampling methods was $99.3 \%$.

NGS from FB samples detected 28 mutations (table 1). Compared with FB analysis alone, the analysis of EBUS-TBNA-TU samples from the 20 subjects considered for comparative analysis revealed four additional mutations. This led to a slight increase of the median (interquartile range) number of detected mutations per patient from $1.0(1.0-2.0)$ to $1.5(1.0-2.3)$, which was not statistically significant $(\mathrm{p}=0.102)$.

The overall median tumour cell content in the samples used for NGS analysis was 48\% (30-70\%) in EBUS-TBNA-TU samples and 46\% (30-60\%) in FB samples.

The material was sufficient for NGS analyses in eight out of 16 tumour-positive EBUS-TBNA-LN samples. Based on the combined analysis of FB and EBUS-TBNA-TU samples, the additional evaluation of LN biopsies detected one additional mutation, which did not change the median number of detected mutations per patient $(\mathrm{p}=0.317)$.

16 out of 20 blood plasma samples were available for ctDNA analysis. Mutations could be detected by NGS in seven subjects. No additional mutations could be detected compared with NGS results from histology samples.

PD-L1 IHC was performed in both EBUS-TBNA-TU and FB samples in 25 subjects, where material was sufficient for PD-L1 staining. Compared with FB alone, EBUS-TBNA detected three additional PD-L1-positive cases (PD-L1 positivity in 64\%). By combining results from EBUS-TBNA and FB samples, a PD-L1 positivity $\geqslant 50 \%$ could be detected in seven $(28 \%)$ out of 25 subjects.

$A L K$ fluorescence in situ hybridisation was negative in all samples from all subjects.

In this study, tumour detection rate and congruency were very high for FB (97\%) and EBUS-TBNA (100\%). Comparing NGS results from FB and EBUS-TBNA-TU in terms of accordance of exon findings, the congruence was $99.3 \%$. These data demonstrate the high reproducibility and reliability of NGS analysis in lung cancer.

Combining tissue sampling by EBUS-TBNA-TU and FB improved the mutation detection rate. The combined sampling elevated the detection rate of PD-L1 positivity.

These data may be of clinical relevance as, according to the approval status, only positive test results allow access to targeted first-line therapy. Data from this study suggest that there is no superiority of one sampling technique regarding NGS results, adjusted mutation frequency and tumour cell content. It remains unclear whether the detection rates may be increased solely by enlarging the sampled tissue or by a greater spread of biopsies over the accessible tumour region $[9,10]$.

A previous study reported a 58\% feasibility for both IHC and NGS in tumour-positive EBUS-TBNA samples [11]. In our study, the feasibility of both tests was $91 \%$. To address the limiting factor of a low yield of tumour DNA, tumour enrichment strategies by demarcating and microdissecting tumour cell areas may reduce false-negative NGS results [12].

To the best of our knowledge, this is the first prospective study in central lung cancer patients comparing NGS data from FB, EBUS-TBNA-TU, EBUS-TBNA-LN and ctDNA with the sequence of endoscopic sampling performed in a randomised order. 
TABLE 1 Detected mutations and corresponding mutation frequency adjusted for tumour cell content (list of subjects where next-generation sequencing analysis was initiated from tumour samples)

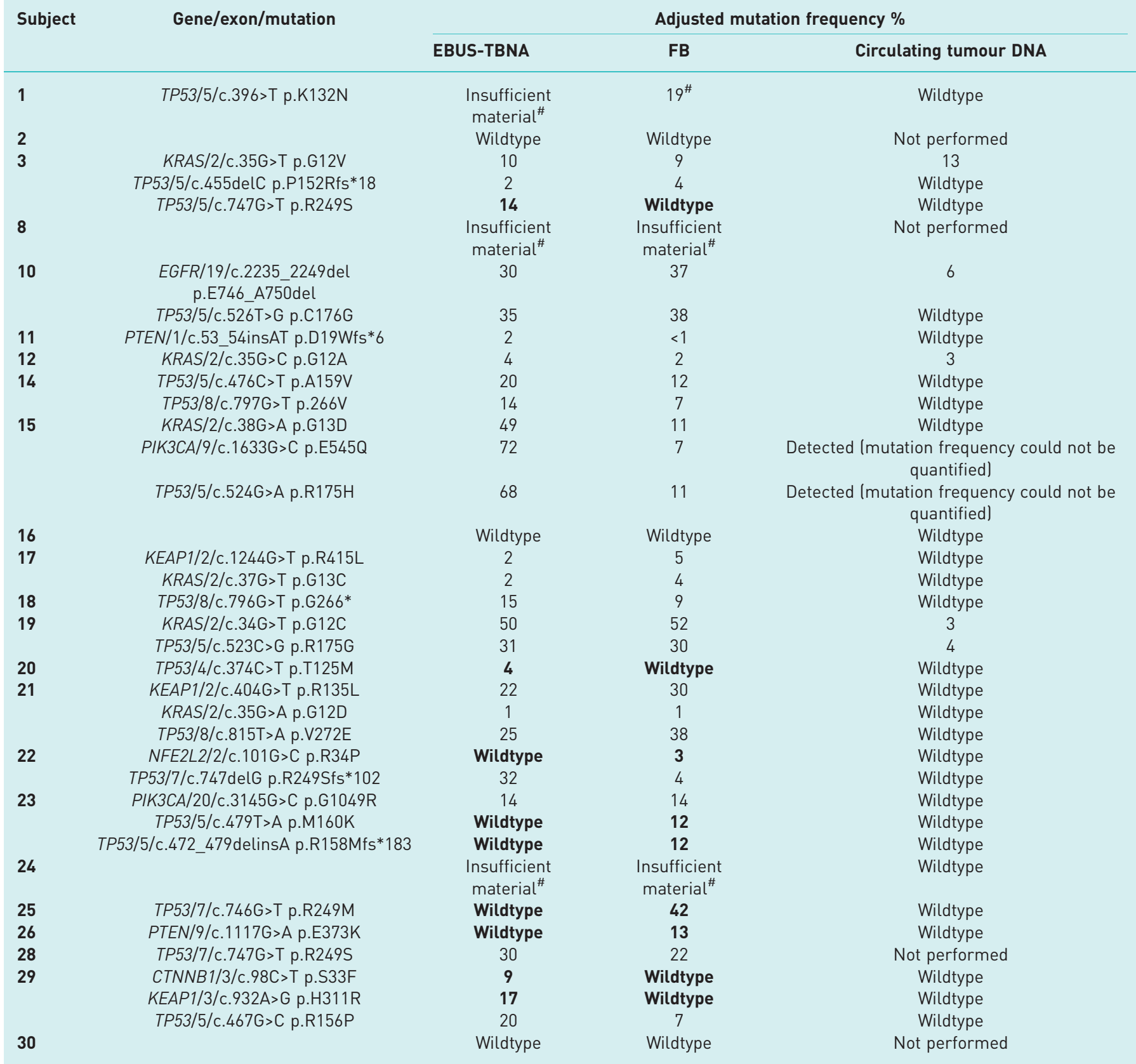

Detected tumour protein alterations with mutations at nucleotide level and change at amino acid level given according to the standard mutation nomenclature in molecular diagnostics [13]. Nonbold: complete agreement of next-generation sequencing results from EBUS-TBNA and FB ( 3 subjects); bold: incomplete agreement of next-generation sequencing results from EBUS-TBNA and forceps biopsy (seven subjects). EBUS-TBNA: endobronchial ultrasound-guided transbronchial needle aspiration; FB: forceps biopsy; TP53: tumour protein 53; KRAS: Kirsten rat sarcoma viral oncogene homologue; EGFR: epidermal growth factor receptor; PTEN: phosphatase and tensin homologue); PIK3CA: phosphatidylinositol-4,5-bisphosphate 3-kinase, catalytic subunit $\alpha$; KEAP1: Kelch-like ECH-associated protein 1; NFE2L2: nuclear factor, erythroid 2-like 2; CTNNB1: catenin- $\beta_{1} .{ }^{*}$ : comparison of EBUS-TBNA and FB results not possible.

Considering all data from this pilot study, a combined FB, EBUS-TBNA-TU and EBUS-TBNA-LN tissue sampling approach may represent a sensitive tool for the detection of oncogenic mutations and PD-L1 positivity. ctDNA analysis should be considered as the last resort. However, the quantity of ctDNA isolated from the plasma samples may be a limiting factor of this approach. 
Our study is limited by the low number of cases. Conclusions must be drawn with caution. As the design is prospective with the sequence of endoscopic sampling performed in a randomised order, the data may be regarded as robust and hypothesis generating.

In central lung cancer, the combination of FB and EBUS-TBNA for tissue sampling may improve the detection rate of oncogenic mutations and PD-L1 positivity. Large multicentre studies are necessary to prove this hypothesis.

Lars Hagmeyer $\oplus^{1,2}$, Stephan Schäfer ${ }^{3}$, Marianne Engels ${ }^{3}$, Jana Fassunke $\odot^{3}$, Anja Pietzke-Calcagnile ${ }^{1}$, Marcel Treml ${ }^{1}$, Simon-Dominik Herkenrath ${ }^{1,2}$, Sandhya Matthes $\oplus^{2}$, Jürgen Wolf ${ }^{4}$, Reinhard Büttner ${ }^{3}$ and Winfried Randerath ${ }^{1,2}$

${ }^{1}$ Institute of Pneumology, University of Cologne, Solingen, Germany. ${ }^{2}$ Hospital Bethanien Solingen, Clinic of Pneumology and Allergology, Center for Sleep Medicine and Respiratory Care, Solingen, Germany. ${ }^{3}$ University of Cologne, Institute of Pathology, Cologne, Germany. ${ }^{4}$ University Hospital of Cologne, Lung Cancer Group Cologne, Department I of Internal Medicine, Cologne, Germany.

Correspondence: Lars Hagmeyer, Hospital Bethanien Solingen, Clinic for Pneumology and Allergology, Center of Sleep Medicine and Respiratory Care, Aufderhöherstraße 169-175, 42699 Solingen, Germany. Email: lars.hagmeyer@klinik-bethanien.de

Received: 4 Jan 2020 | Accepted after revision: 6 May 2020

Support statement: This research was funded by Astra Zeneca. Funding information for this article has been deposited with the Crossref Funder Registry.

Author contributions: L. Hagmeyer: Substantial contributions to conception and design, analysis and interpretation of data, drafting the article and finalising the version to be published taking responsibility for the integrity of the work as a whole, from inception to published article. S. Schäfer: Acquisition of data, analysis and interpretation of data, revising the article critically for important intellectual content and final approval of the version to be published. M. Engels: Acquisition of data, analysis and interpretation of data, revising the article critically for important intellectual content and final approval of the version to be published. J. Fassunke: Acquisition of data, analysis and interpretation of data, revising the article critically for important intellectual content and final approval of the version to be published. A. Pietzke-Calcagnile: Acquisition of data, analysis and interpretation of data, drafting statistics section and revising the article and final approval of the version to be published. M. Treml: Acquisition of data, analysis and interpretation of data, drafting statistics section and revising the article and final approval of the version to be published. S-D. Herkenrath: Acquisition of data, analysis and interpretation of data, drafting statistics section and revising the article and final approval of the version to be published. S. Matthes: Acquisition of data, analysis and interpretation of data, revising the article and final approval of the version to be published. J. Wolf: Acquisition of data, analysis and interpretation of data, revising the article critically for important intellectual content and final approval of the version to be published. R. Büttner: Acquisition of data, analysis and interpretation of data, revising the article critically for important intellectual content and final approval of the version to be published. W. Randerath: Substantial contributions to conception and design, analysis and interpretation of data, drafting the article and finalising the version to be published.

This study is registered at www.clinicaltrials.gov with identifier number NCT03017183. Individual participant data will not be available. Additional related documents will not be available.

Conflict of interest: L. Hagmeyer reports grants from Astra Zeneca, during the conduct of the study; grants and personal fees from Roche, grants and personal fees from Böhringer Ingelheim, personal fees from Lilly, grants from MSD, outside the submitted work. S. Schäfer has nothing to disclose. M. Engels has nothing to disclose. J. Fassunke has nothing to disclose. A. Pietzke-Calcagnile has nothing to disclose. M. Treml has nothing to disclose. S-D. Herkenrath has nothing to disclose. S. Matthes has nothing to disclose. J. Wolf reports personal fees from Abbvie, personal fees from AstraZeneca, personal fees from Blueprint, grants and personal fees from BMS, personal fees from Boehringer, personal fees from Chugai, personal fees from Ignyta, grants and personal fees from Jannsen, personal fees from Lilly, personal fees from Loxo, grants and personal fees from MSD, grants and personal fees from Novartis, grants and personal fees from Pfizer, personal fees from Roche, personal fees from Takeda, outside the submitted work. R. Büttner has nothing to disclose. W. Randerath reports grants from Astra Zeneca, during the conduct of the study.

\section{References}

1 Kalemkerian GP, Narula N, Kennedy EB, et al. Molecular testing guideline for the selection of patients with lung cancer for treatment with targeted tyrosine kinase inhibitors: American Society of Clinical Oncology Endorsement of the College of American Pathologists/International Association for the Study of Lung Cancer/Association for Molecular Pathology Clinical Practice Guideline Update. J Clin Oncol 2018; 36: 911-919.

2 Hanna N, Johnson D, Temin S, et al. Systemic therapy for stage IV non-small-cell lung cancer: American Society of Clinical Oncology Clinical Practice Guideline Update. J Clin Oncol 2017; 35: 3484-3515.

3 Du Rand IA, Blaikley J, Booton R, et al. British Thoracic Society guideline for diagnostic flexible bronchoscopy in adults: accredited by NICE. Thorax 2013; 68: Suppl. 1, i1-i44.

4 Scheel AH, Dietel M, Heukamp LC, et al. [Predictive PD-L1 immunohistochemistry for non-small cell lung cancer current state of the art and experiences of the first German harmonization study]. Pathologe 2016; 37: 557-567. 
5 Scheel AH, Dietel M, Heukamp LC, et al. Harmonized PD-L1 immunohistochemistry for pulmonary squamous-cell and adenocarcinomas. Mod Pathol 2016; 29: 1165-1172.

6 Peifer M, Fernandez-Cuesta L, Sos ML, et al. Integrative genome analyses identify key somatic driver mutations of small-cell lung cancer. Nat Genet 2012; 44: 1104-1110.

7 George J, Lim JS, Jang SJ, et al. Comprehensive genomic profiles of small cell lung cancer. Nature 2015; 524: $47-53$.

8 Clinical Lung Cancer Genome Project, Network Genomic Medicine. A genomics-based classification of human lung tumors. Sci Transl Med 2013; 5: 209ra153.

9 Bassanelli M, Sioletic S, Martini M, et al. Heterogeneity of PD-L1 expression and relationship with biology of NSCLC. Anticancer Res 2018; 38: 3789-3796.

10 McGranahan N, Swanton C. Clonal heterogeneity and tumor evolution: past, present, and the future. Cell 2017; 168: 613-628.

11 Rooper LM, Nikolskaia O, Carter J, et al. A single EBUS-TBNA procedure can support a large panel of immunohistochemical stains, specific diagnostic subtyping, and multiple gene analyses in the majority of non-small cell lung cancer cases. Hum Pathol 2016; 51: 139-145.

12 Layfield LJ, Roy-Chowdhuri S, Baloch Z, et al. Utilization of ancillary studies in the cytologic diagnosis of respiratory lesions: The Papanicolaou Society of Cytopathology Consensus recommendations for respiratory cytology. Diagn Cytopathol 2016; 44: 1000-1009.

13 Ogino S, Gulley ML, den Dunnen JT, et al. Standard mutation nomenclature in molecular diagnostics: practical and educational challenges. J Mol Diagn 2007; 9: 1-6. 